\title{
A 10 KW IRFEL DESIGN FOR JEFFERSON LAB*
}

\author{
D. Douglas, S. V. Benson, G. Biallas, J. Boyce, H. F. Dylla, R. Evans, A. Grippo, J. Gubeli, \\ K. Jordan, G. Krafft, R. Li, J. Mammosser, L. Merminga, G. R. Neil, L. Phillips, J. Preble, \\ M. Shinn, T. Siggins, R. Walker, and B. Yunn, \\ Thomas Jefferson National Accelerator Facility, Newport News, VA 23606, USA
}

\section{Abstract}

Recent work at Jefferson Lab has demonstrated the viability of same-cell energy recovery as a basis for a high average power free-electron laser (FEL) [1]. We are now extending this technique to lase at average powers in excess of $10 \mathrm{~kW}$ in the infrared. This upgrade will also produce over $1 \mathrm{~kW}$ in the UV and generate high brightness Thomson back-scattered X-rays. The power increase will be achieved by increasing the electron beam energy by a factor of four, and the beam current and the FEL design efficiency by a factor of two. Utilization of a nearconcentric optical cavity is enabled by the use of very low loss state-of-the-art coatings. The FEL will be placed in the return leg of the electron beam transport, giving a machine footprint quite similar to that of the existing $1 \mathrm{~kW}$ IR device.

Some features of the Upgrade are straightforward extensions of those in the present $1 \mathrm{~kW}$ design; others break new ground and present new challenges. These will be described. The required electron beam parameters and the laser performance estimates will be summarized. Changes required in the electron beam transport will be outlined and the optical cavity design briefly reviewed.

\section{EXPERIENCE GAINED FROM THE INFRARED DEMONSTRATION LASER}

The Jefferson Lab IR Upgrade FEL is an evolutionary derivative of the JLab IR Demo FEL. It thus retains the approach used in the earlier machine - that of a low peak, high average power wiggler-driven optical cavity resonator with an energy recovering SRF linear accelerator driver operating at high repetition rate. The $10 \mathrm{~kW}$ design goal will be achieved via an increase in both drive beam power (doubled current and quadrupled energy) and FEL extraction efficiency (from $0.5 \%$ to $1 \%$ ). The design builds on experience gained with the Infrared Demonstration FEL (IR Demo FEL) now operating at Jefferson Lab.

Primary electron beam specifications for the IR Demo and Upgrade are listed in Table 1. Several features of the IR Demo have influenced the design for the Upgrade:

- The injector produces a beam with enough brightness at $135 \mathrm{pC}$ to drive the FEL with adequate lifetime (nearly $700 \mathrm{C}$ - over 40 hours - at high current [2]). Initial experience demonstrates that performance at this higher charge is acceptable.
Table 1: IR Demo and Upgrade Parameters

\begin{tabular}{|c|c|c|c|}
\hline Parameters & Demo & Upgrade & $\begin{array}{c}\text { Achieved } \\
(6 / 2001)\end{array}$ \\
\hline energy $(\mathrm{MeV})$ & $35-48$ & $80-210$ & $20-48$ \\
\hline $\mathrm{I}_{\mathrm{ave}}(\mathrm{mA})$ & 5 & 10 & 5 \\
\hline $\begin{array}{c}\text { FEL rep. rate } \\
(\mathrm{MHz})\end{array}$ & $\begin{array}{c}18.75- \\
75\end{array}$ & $3.9-125$ & $18.75-75$ \\
\hline $\mathrm{Q}_{\text {bunch }}(\mathrm{pC})$ & 60 & $\begin{array}{c}135 @ 75 \\
\mathrm{MHz}\end{array}$ & $\begin{array}{c}135 @ 37.5 \\
\mathrm{MHz}\end{array}$ \\
\hline $\begin{array}{c}\text { bunch } \sigma_{1} \\
\text { (psec) }\end{array}$ & 0.4 & $\begin{array}{c}0.2 @ 135 \\
\mathrm{pC}\end{array}$ & $0.4 @ 60$ pC \\
\hline $\mathrm{I}_{\text {peak }}(\mathrm{A})$ & 60 & $\begin{array}{l}270 @ \\
135 \mathrm{pC} \\
\end{array}$ & $60 @ 60$ pC \\
\hline$\sigma_{\delta \mathrm{p} / \mathrm{p}}(\%)$ & $0.5 \%$ & $0.5 \%$ & $\begin{array}{c}0.25 \% @ 60 \\
\text { pC } \\
\end{array}$ \\
\hline$\varepsilon_{\mathrm{N}}(\mathrm{mm}-\mathrm{mrad})$ & $<13$ & $<30$ & $\begin{array}{l}5-10 @ 60 \mathrm{pC} \\
25 @ 135 \mathrm{pC} \\
\end{array}$ \\
\hline$\eta_{\mathrm{FEL}}$ & $0.5 \%$ & $1 \%$ & $>1 \%$ \\
\hline $\begin{array}{l}\Delta \mathrm{E}_{\text {full }} \text { after } \\
\text { FEL }\end{array}$ & $5 \%$ & $10 \%$ & $6-8 \%$ \\
\hline
\end{tabular}

- Beam loss at full energy can be held to quite low values. Measured total losses for energies in excess of $15 \mathrm{MeV}$ are less than $0.1 \%$ and single point losses inferred from beam line activation and radiation measurements near the wiggler are much less than $100 \mathrm{nA}$. The apertures in the Upgrade have been enlarged to accommodate larger beam envelope functions and emittance. We anticipate that total losses should be much less than $0.1 \%$.

- Operation at the peak of the power vs. cavity length curve is possible with no instabilities. This has been demonstrated in the IR Demo. Because the threshold for the RF/FEL instability with feedback on is on the order of $1 \mathrm{~A}$ of beam current, operation at $5 \mathrm{~mA}$ of current is quite stable [3].

- The near-concentric cavity design used in the IR Demo can be used at even higher power without power limitations. The power limit is now known to be due to heating-induced aberrations [4], which arise when the power absorbed in a sapphire output coupler exceeds $40 \mathrm{~W}$ at $3 \mu \mathrm{m}$. For the IR Demo, this occurs at over $4 \mathrm{~kW}$ of laser power. FEL efficiency limits the power before this limit is reached. When the laser efficiency vs. bunch charge is 
measured, it is found that the efficiency is still increasing at the highest charge accelerated.

- Sextupoles can be used to linearize the bunching and debunching processes so that the peak current at the FEL is very high and the energy spread at the final dump is small. This is done routinely in the IR Demo FEL to minimize the energy spread at the final dump; the momentum spread has been compressed from $8 \%$ full width at $48 \mathrm{MeV}$ down to $\sim 1 \%$ full width at $9.5 \mathrm{MeV}$. For very large induced energy spread one can use octupoles to linearize the transport over an even larger range.

\section{2. $10 \mathrm{KW}$ IRFEL DESIGN SOLUTION}

Figure 1 illustrates a machine design solution incorporating the above experience. It comprises a $10 \mathrm{MeV}$ injector, a linac consisting of three Jefferson Lab cryomodules generating 80 to $210 \mathrm{MeV}$ of energy gain, and a recirculator. The latter provides beam transport to, and phase space conditioning of, the accelerated electron beam for the FEL and then returns and prepares the drive beam for energy recovery in the linac.

The injector is a direct upgrade of the IR Demo injector [5] from $5 \mathrm{~mA}$ at $10 \mathrm{MeV}$ to $10 \mathrm{~mA}$ at $10 \mathrm{MeV}$. The current will be doubled by an increase of the single bunch charge from $67 \mathrm{pC}$ to $135 \mathrm{pC}$ while maintaining the 75 $\mathrm{MHz}$ repetition rate. Adequate source and injector performance have already been demonstrated at the elevated bunch charge (at a source-limited repetition rate of 37.5 $\mathrm{MHz}$ ) [2] and will be characterized at full power when a higher power RF drive system is installed. Planned improvements in the standoff capability of the photocathode may allow even brighter source performance.

The linac comprises three Jefferson Lab cryomodules; the first and third incorporate conventional five-cell CEBAF cavity designs, and the central module is based on new seven-cell JLAB cavities [6]. The lower gradient five-cell modules are placed at the end of the accelerator to limit RF focussing effects on the low energy injected and energy-recovered beams. The beam is accelerated (energy recovered) off crest (off trough) so as to impose a phase energy correlation on the longitudinal phase space. This is used in subsequent transport to longitudinally match the beam to the required phase space at the wiggler (dump).

The first segment of the recirculator provides transport of the drive beam from the linac to the FEL. Immediately following the linac, a small chicane separates the energy recovered and full energy beams, dumping the low energy and directing the high energy to a six quad telescope that transversely matches it to a recirculation arc. This arc, based a Bates endloop [7], transports the beam to the machine backleg and longitudinally conditions it in preparation for the FEL interaction. The arc first and second order momentum compactions are set to rotate the bunch (which has been slewed by off-crest acceleration) upright at the wiggler and to eliminate phase space curvature, producing a short bunch and high peak current. Downstream of the arc, a FODO transport line conveys the beam to the FEL insertion.

The FEL insertion commences with a chicane around the upstream end of a $32 \mathrm{~m}$ high-power optical cavity. This provides clearance between electron beam and optical components and generates momentum compaction for a final compression of the bunch length. A six-quad telescope transversely matches the beam to the wiggler; a second telescope downstream of the wiggler provides a match from the wiggler to the energy recovery transport.

The energy recovery transport consists of a second Bates-style endloop (returning the beam to the linac) followed by a six quad telescope. The beam is matched to the arc by the second telescope of the FEL insertion; the energy recovery telescope matches beam envelopes from the arc to the linac acceptance. Trim quads, sextupoles, and octupoles in the arc adjust momentum compactions through third order to longitudinally rotate the short, very large momentum spread bunch and adjust its curvature and torsion in preparation for energy recovery. Because energy recovery occurs off-trough, the imposed phaseenergy correlations are selected to generate energy compression during energy recovery, yielding a long, low momentum spread bunch at the dump.

All apertures in the energy recovery loop are chosen to allow very large energy spread to be transported without loss to the energy recovery dump. Experience with the the IR Demo shows the Bates endloop to be a very robust design; at least $8 \%$ energy spread beam has been transported cleanly to the end-of-line dump. Simulations indicate that the Upgrade will tolerate an initial energy spread of $15 \%$ - compressing it to a final spread of order $\pm 1 \%$ despite the larger ratio of final to initial energy (Figure 2).

\section{WIGGLER AND OPTICAL RESONATOR}

To provide flexibility and allow operation over a range of wavelengths, an optical klystron was chosen for the wiggler. This allows the extraction efficiency to be varied continuously over a large range. The optical klystron has two wigglers of 12 periods each separated by a dispersion section with 40 periods of path-length delay at the highest wiggler strength. The wiggler period is $20 \mathrm{~cm}$ and the maximum $\mathrm{rms} \mathrm{K}^{2}$ is 16 . The wavelength can thus be tuned over a large range without resetting the accelerator energy. One can, for example, tune from 2 to 25 microns at a fixed energy of $130 \mathrm{MeV}$. Each wiggler will be shunted so that its field can be shifted with respect to that in the other; this may allow higher efficiency without larger exhaust energy spread [8].

The optical resonator will be centered at the point of maximum angle in the dispersion section. The laser will be operated with a short electron bunch and weak dispersion section for production of fsec X-ray pulses. Thomson 
backscattered X-rays produced at the collision point will be directed upward and deflected out of the beam chamber downstream of the FEL using a scattering crystal. The X-ray bunches should have an rms bunch length of approximately $200 \mathrm{fsec}$.

The optical resonator is similar to the one used in the IR Demo FEL. To accommodate a longer wiggler, the resonator length has been increased to $32 \mathrm{~m}$. Changes in waist size with mirror heating will be eliminated by using a deformable high reflector, which with match a change in the output coupler radius of curvature with an equal and opposite change. The output coupling will also be increased. With these changes, the maximum intensity on the mirrors will be similar to that demonstrated in the IR Demo FEL. The drawback to this technology is that the waist position will change as the power increases; since this shift in the position is much smaller than the Rayleigh range, this should not affect the lasing.

Gain and power calculated for IR Upgrade parameters are shown in Figure 3 for operation of the optical klystron as a 25 period wiggler. Both power and gain are very weakly dependent on the emittance and energy spread.

\section{BEAM DYNAMICS AND COLLECTIVE PHENOMENA}

Beam dynamics concerns include configuration of the electron beam for the FEL interaction and the impact of collective effects. To achieve the higher peak current in the Upgrade, the longitudinal phase space can be managed as in the IR Demo [9]. Adiabatic damping will reduce the bunch intrinsic relative momentum spread (the "thickness" of the slewed phase ellipse) after the module; when rotated upright at the wiggler, the bunch will then be shorter. Recirculator sextupoles will be used in concert to compensate both lattice and RF-waveform induced curvature of the longitudinal phase space.

Numerous collective phenomena can compromise system performance. These include space charge, wakefield effects, BBU, CSR, and the FEL/RF interaction. The longitudinal manipulations of the IR Demo minimize many of these effects since the bunch length is long in most of the machine. Space charge effects are significant primarily in the injector; at energies over $25 \mathrm{MeV}$, IR Demo experience indicates system behavior will be insensitive to space charge driven phenomena. To date, adequate performance at the design single bunch charge of $135 \mathrm{pC}$ has been demonstrated. Further PARMELA and empirical analysis of machine performance, particularly in the injector, will occur as the project continues [10].

A preliminary analysis of wakefield effects [10] suggests an impedance budget based on (2 in. aperture) IR Demo components will yield marginally acceptable beam performance at the FEL. We will use 3 in. apertures in the transport from the linac to the wiggler to allow margin in the Upgrade design.
Preliminary results extracted from a rudimentary CSR simulation using a one-dimensional longitudinal wake model suggest Upgrade performance will not be compromised by CSR effects. Future work will involve the application of a more sophisticated CSR model to the design; this will allow comprehensive analysis of CSR effects in the system, including dependence on details of charge distribution within the bunch [11].

Investigation of BBU effects suggests instability thresholds will significantly exceed anticipated operating currents [12]. As mentioned above, the RF/FEL instability is well handled by the RF control systems. The threshold for the instability should be near 1 A [3].

\section{PROJECT STATUS}

US Navy funding for the project has been received. A machine design has been generated and has gone into engineering. The IR Demo will be shut down in December 2001 and commissioning of the upgraded machine will start in late 2002. Funding is also approved for a bypass allowing operation in the UV with an undulator similar to the Undulator A used at Argonne.

Since the lattice is very robust, it is possible to replace the two five-cell cryomodules with larger aperture, upgraded seven-cell cryomodules and achieve even higher power. With an upgraded injector providing up to 100 $\mathrm{mA}$ and a wiggler optimized for shorter wavelength operation, the system can be upgraded in a straightforward manner to exceed $100 \mathrm{~kW}$ of laser power in the near infrared.

\section{ACKNOWLEDGMENTS}

Work supported by the U. S. Department of Energy under contract DE-AC05-84-ER40150, the Office of Naval Research, the Commonwealth of Virginia, and the Laser Processing Consortium.

\section{REFERENCES}

[1] G. R. Neil et al., Phys. Rev. Lett., 84, (2000) 662.

[2] T. Siggins et al., FEL2000, Durham, NC, Aug. 2000.

[3] L. Merminga et al., FEL'99, Hamburg, Aug. 1999.

[4] S. Benson, FEL2001, Darmstadt, FRG, Aug. 2001.

[5] D. Engwall et al., PAC'97, pp. 2693-5, Vancouver, May 1997.

[6] J. R. Delayen et al., PAC'99, pp. 934-6, New York, 29 March-2 April 1999.

[7] J. Flanz et al., Nucl. Inst. Meth. A241:325-33 (1985).

[8] D. A. Jaroszynski et al., Nucl. Inst. and Meth. A358 (1995) 228.

[9] D. Douglas, Linac 2000, Monterey, CA, Aug. 2000.

[10] L. Merminga and B. Yunn, FEL2000, Durham, NC, Aug. 2000.

[11]R. Li, PAC'99, pp. 118-22, New York, 29 March-2 April 1999; R. Li, these proceedings.

[12] L. Merminga et al., PAC'99, pp. 1180-2, New York, 29 March-2 April 1999. 


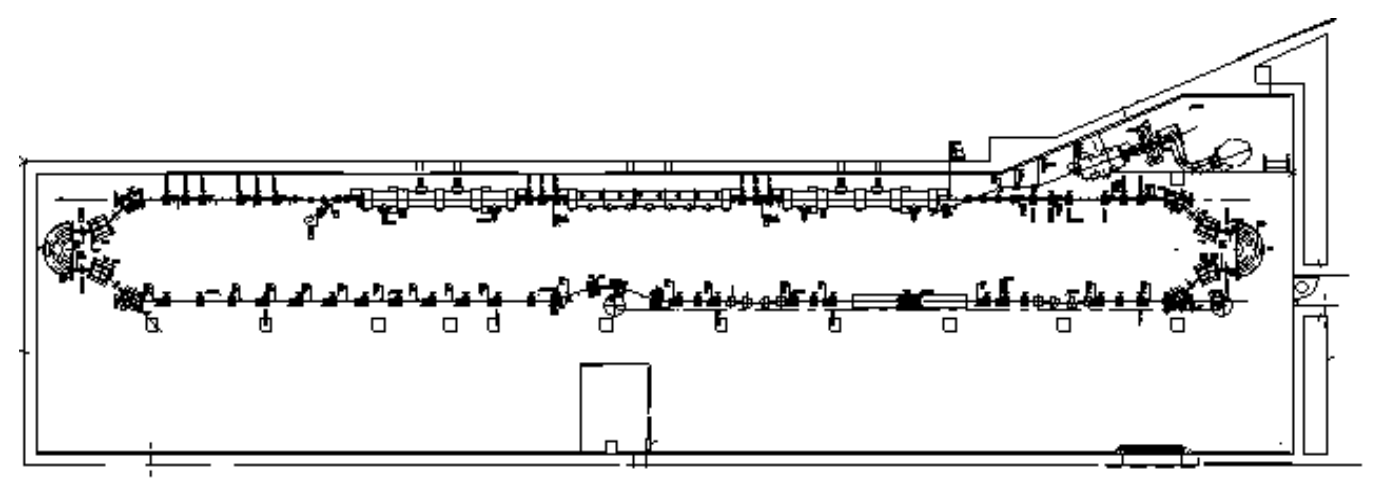

Figure 1. Layout of the IR Upgrade. The electron beam is accelerated in two SRF cavities and injected into a 3cryomodule accelerator. The beam is turned 180 degrees in a Bates style bend and matched into the wiggler across a buncher chicane. The exhaust beam is turned 180 degrees and reinjected into the accelerator shifted by 180 degrees in $\mathrm{RF}$ phase with respect to the accelerated beam. Weak chicanes merge the injected and energy recovered beams and separate the accelerated and decelerated beams.
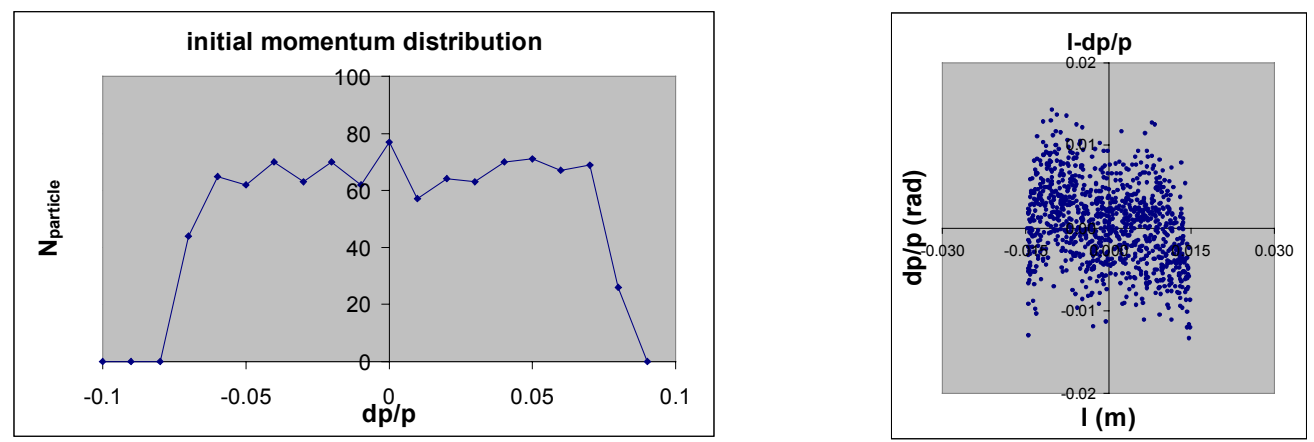

Figure 2: Simulation of energy recovery; momentum distribution at wiggler (left) and longitudinal phase space at dump (right) during high power lasing. The initial $15 \%$ relative energy spread is compressed to $\sim 1 \%$ during energy recovery.

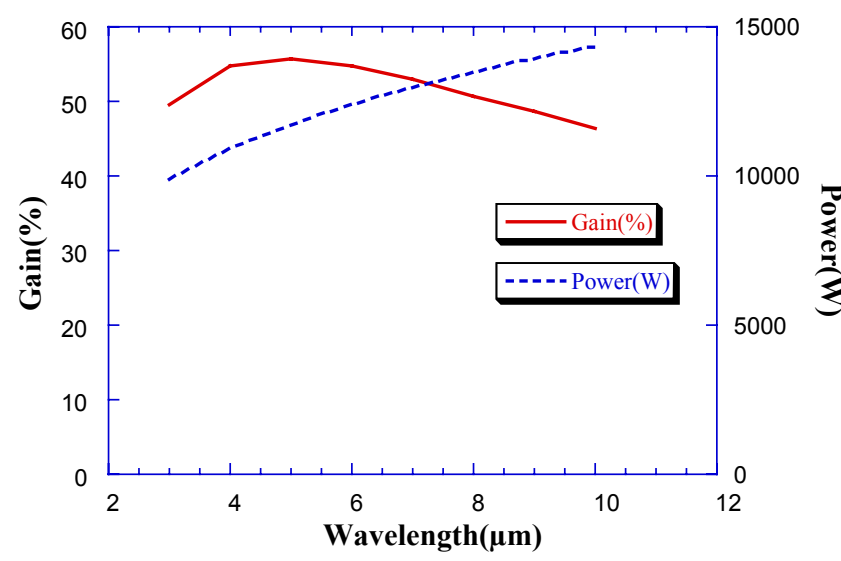

Figure 3: Gain and Power calculated for the IR Upgrade when the optical klystron is operated with minimum dispersion 